\title{
Robert Hooke's Seminal Contribution to Orbital Dynamics
}

\author{
Michael Nauenberg*
}

\begin{abstract}
During the second half of the seventeenth century, the outstanding problem in astronomy was to understand the physical basis for Kepler's laws describing the observed orbital motion of a planet around the Sun. Robert Hooke (1635-1703) proposed in the middle 1660s that a planet's motion is determined by compounding its tangential velocity with its radial velocity as impressed by the gravitational attraction of the Sun, and he described his physical concept to Isaac Newton (1642-1726) in correspondence in 1679. Newton denied having heard of Hooke's novel concept of orbital motion, but shortly after their correspondence he implemented it by a geometric construction from which he deduced the physical origin of Kepler's area law, which later became Proposition I, Book I, of his Principia in 1687. Three years earlier, Newton had deposited a preliminary draft of it, his De Motu Corporum in Gyrum (On the Motion of Bodies), at the Royal Society of London, which Hooke apparently was able to examine a few months later, since shortly thereafter he applied Newton's construction in a novel way to obtain the path of a body under the action of an attractive central force that varies linearly with the distance from its center of motion (Hooke's law). I show that Hooke's construction corresponds to Newton's for his proof of Kepler's area law in his De Motu. Hooke's understanding of planetary motion was based on his observations with mechanical analogs. I repeated two of his experiments and demonstrated the accuracy of his observations. My results thus cast new light on the significance of Hooke's contributions to the development of orbital dynamics, which in the past have either been neglected or misunderstood.
\end{abstract}

Key words: Robert Hooke; Isaac Newton; astronomy; orbital motion.

\section{Introduction}

One of the most fascinating questions in the history of science concerns the role that Robert Hooke (1635-1703) played in the development of dynamics and the theory of gravitation during the seventeenth century, which culminated in Isaac Newton's masterpiece, the Principia in 1687. ${ }^{1}$ Hooke was one of the most prolific and inventive scientists of all times, and he made fundamental contributions to virtual all branches of science; ${ }^{2}$ one third of the fifteen volumes of Robert T. Gunther's Early Science in Oxford are dedicated to his work. ${ }^{3}$

Despite Hooke's profound influence, however, particularly on Newton's development of orbital dynamics, he was nearly completely forgotten after his death in 1703 ,

* Michael Nauenberg is Professor Emeritus of Physics at the University of California, Santa Cruz. His primary research has been in theoretical physics, but he also has written several articles and co-edited a book on the historical development of dynamics by Huygens, Newton, and Hooke. 
and remained unknown until around the turn of the 20th century.* Ernst Mach, in his influential book of 1883, The Science of Mechanics, devoted only a few lines to Hooke, although he perceptively stated that "Hooke really approached nearest to Newton's conception, though he never completely reached the latter's altitude of view." ${ }^{4}$ Ten years later, W.W. Rouse Ball published some of the correspondence between Hooke and Newton (1642-1726), 5 and the subsequent discovery of two additional letters of Hooke, which were published by Jean Pelseneer and Alexandre Koyré, ${ }^{6}$ initiated a reappraisal of Hooke's contributions to mechanics that continues to the present time. ${ }^{7}$ René Dugas, in contrast to Mach, recognized Hooke's crucial role in his book of 1958, Mechanics in the Seventeenth Century, ${ }^{8}$ but some recent accounts of the development of mechanics still ignore Hooke's significant contributions. Most physicists and mathematicians still remain unaware of them, as can be seen by reading modern textbooks or journals that cover classical mechanics, where Hooke is mentioned only in connection with his eponymous law of elasticity. ${ }^{9}$

During the past few years, however, a number of books and articles have appeared that describe Hooke's many contributions to science, and also his major role in the reconstruction of London after the Great Fire of $1666 .{ }^{10}$ There now appears to be consensus among historians of science that Hooke's physical explanation for the orbital motion of planets - "compounding the celestiall motions of the planetts of a direct motion by the tangent $\&$ an attractive motion towards the centrall body" 11 - had an influence on Newton's work that culminated in the publication of his Principia. Hooke communicated his ideas on orbital motion to Newton in a letter in 1679 and, according to one of Newton's most outstanding biographers, Richard S. Westfall:

Newton's papers reveal no similar understanding of circular motion before this letter. Every time he had considered it, he had spoken of a tendency to recede from the center, what [Christiaan] Huygens [1629-1695] called centrifugal force; and like others who spoke in such terms, he had looked upon circular motion as a state of equilibrium between two equal and opposing forces, one away from the center and one toward it. Hooke's statement treated circular motion as a disequilibrium in which an unbalanced force deflects a body that would otherwise continue in a straight line. It was not an inconsiderable lesson for Newton to learn. ${ }^{12}$

But there is considerable confusion in the literature as to exactly what the "lesson" was that Newton learned from Hooke. In a letter to Edmond Halley (1656-1742) on June $20,1679,{ }^{13}$ Newton vehemently denied that he had learned anything form Hooke, although he admitted, in one of his unpublished autobiographical manuscripts, that:

In the end of the year 1679 in answer to a Letter from Dr Hook ... I found now that whatsoever was the law of the forces $\mathrm{w}^{\mathrm{ch}} \mathrm{kept}$ the Planets in their Orbs, the areas described by a Radius drawn from them to the Sun would be proportional to the times in $\mathrm{w}^{\mathrm{ch}}$ they were described. And by the help of these two Propositions I found that their Orbs would be such Ellipses as Kepler had described. ${ }^{14}$

* Symtomatic of the neglect of Hooke and his legacy is that he is virtually alone among the great scientists of the past for whom no extant portrait exists. 
To understand the influence of Hooke's concept of orbital motion on Newton, we must know how Newton viewed orbital dynamics prior to Hooke's letter to him in 1679. Newtonian scholars commonly conclude that Newton's crucial step thereafter was to switch from a traditional view of circular motion as giving rise to a centrifugal force or tendency to recede from the center, to the concept of a centripetal force directed toward the center.* As I have shown, however, this is misleading in view of Newton's already profound albeit incomplete understanding of orbital motion at that time. ${ }^{15}$

In the following sections, I first describe Newton's early development of orbital dynamics as based on his "Waste Book" of 1664 and on his letter of December 13, 1679, to Hooke. I then present Hooke's formulation of the physical principles of orbital motion for central forces and analyze his recently discovered diagram of 1685 for the motion of a body under the action of a central force that varies linearly with the distance. ${ }^{16}$ I show graphically that it implements his dynamical principles in a way very similar to Newton's description of his proof of Kepler's area law in his De Motu Corporum in Gyrum (On the Motion of Bodies) of $1684,{ }^{17}$ the preliminary draft of his Principia. I next present further background on the development of Hooke's physical ideas, which were based on mechanical analogs. I repeated Hooke's experiments on a conical pendulum and on a ball rolling inside an inverted cone, finding both to agree with Hooke's observations. I close with a summary and conclusions.

\section{Newton's Theory of Orbital Motion Prior to Hooke's Letter of 1679}

René Descartes (1596-1650) illustrated the traditional view of circular motion in 1644 by considering a stone rotating in a sling (figure 1$).{ }^{18}$ If the stone is freed at point $A$, then it would move along the tangent to the circle from point $A$ to point $C$, but instead the sling constrains it to move along a circular path to point $B$. The "tendency" of the stone to recede radially is felt by the person's hand at the center, since the stone exerts a force that depends on its weight and velocity and on the length of the sling. The magnitude of this force was not known quantitatively until Huygens and somewhat later Newton showed that it is proportional to the radial acceleration, ${ }^{19}$ which is equal to the square of the velocity of the stone divided by the length of the sling.

Prior to 1679 , Newton based his description of the orbital motion of a body under the action of a central force on a generalization of the properties of circular motion, ${ }^{20}$ compounding its orbital velocity along the tangent with a change of velocity perpendicular to this direction, whereas Hooke considered its total change in velocity directed toward the center of force. Newton thought of orbital motion in this way at least as early as 1664, when he wrote in his "Waste Book" that, "If the body ... moved in an

* For example, I. Bernard Cohen claims that, "With regard to circular or general curved orbital motion, ... Newton was still [in 1679] under the spell of Cartesian physics and was thinking about centrifugal rather than centripetal forces, or rather endeavors. Thus, in the case of orbital motion, he was contemplating a radial displacement that was tangentially directed outward, away from the center, rather than the more fruitful concept of a radial displacement inward." See Cohen and Whitman, The Principia (ref. 1), pp. 76-77. 


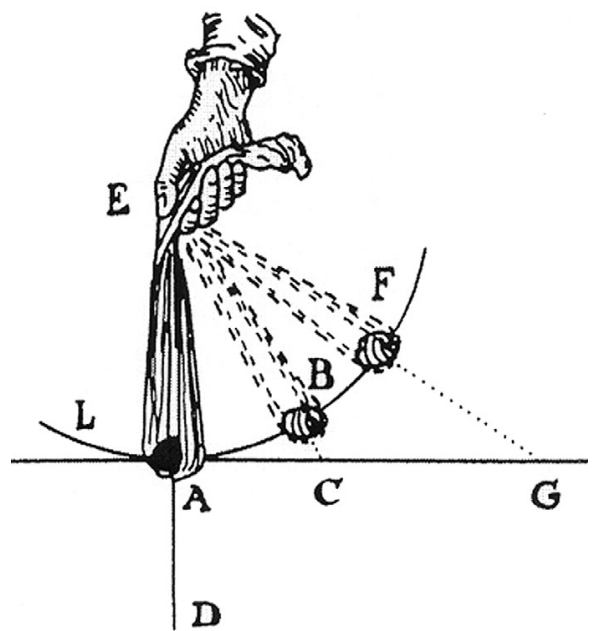

Fig. 1. Descartes's illustration of 1644 of the traditional view of circular motion as a "tendency to recede from the center." Source: Descartes, Principles of Philosophy (ref. 16), plate ii, p. 290.

Ellipsis, then its force in each point (if its motion [velocity] in that point bee given) may bee found by a tangent circle of Equall crookednesse with that point of the Ellipsis." 21

Newton equated force with acceleration, and by a "circle of equal crookednesse" (his word for curvature) he meant the osculating circle (as Gottfried Leibniz [1646-1716] called it thirty years later) at a point on the ellipse. Newton evidently was applying his concept of radial acceleration for circular motion, with the radius being the radius of curvature at a point on the ellipse. For noncircular motion, there is a component of acceleration perpendicular to the orbit, but then the center of curvature and the center of force do not coincide and the velocity along the orbit is not constant, because there is a component of the force tangential to the orbit that produces a tangential acceleration. Thus, Newton required, as he stated, that the motion or velocity at a point on the ellipse "bee given." Newton discovered how to calculate this velocity by Kepler's area law (or what we now call conservation of angular momentum), which was the essential missing link in his analysis, only after he received Hooke's letter of 1679.

Further evidence that the lesson that Newton learned from Hooke was not simply to switch from a centrifugal to a centripetal representation of force, as some Newtonian scholars have claimed, can be found in a letter that Newton wrote to Hooke on December 13, 1679.22 In its corner he drew a diagram (figure 2) that shows he was able to compute "by points quam proxime" (approximately) the orbit of a body under the action of a constant central force (see Appendix). His text indicates that he also understood very well the changes that would occur in this orbit if the force increases toward the center, as I have discussed in detail elsewhere. ${ }^{23}$ Moreover, Newton continued to argue in terms of his concept of centrifugal force long after 1679. For example, following some arguments with John Flamsteed (1646-1719), the first Astronomer Royal, 


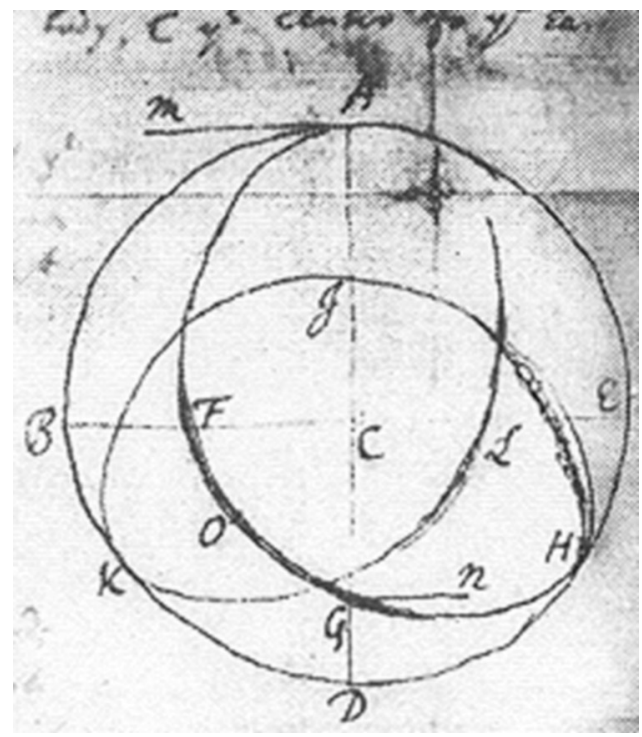

Fig. 2. Newton's diagram in his letter to Hooke of December 13, 1679, showing the orbit of a body moving under the action of a constant attractive force directed toward the center $C$. The body is launched at $A$ with a velocity in the direction $A m$ and moves along the trajectory $A F O G H I K L$. There is an error in this diagram in the magnitude of the angle between the successive "apogees" $A, H$, and $K$, leading historians of science to believe that Newton did not know how to calculate this orbit at this time, but I have shown that Newton made this error inadvertendly when he drew this diagram and did not err in his actual computation of the orbit; see Nauenberg, "Newton's Early Computational Method" (ref. 14). Courtesy of the British Library.

Newton explained in a letter to James Crompton* in April 1681 that the orbit of a comet around the Sun is determined by "the vis centrifuga [centrifugal force] at $C$ [perihelium] overpow'ring the attraction \& forcing the Comet there notwithstanding the attraction, to begin to recede from ye Sun." 24 In fact, Newton never abandoned his concept of orbital dynamics based upon his curvature approach, ${ }^{25}$ and in Book 3, Proposition 28, of his Principia he applied it in combination with Kepler's area law to obtain a remarkable solution of the effect of solar perturbation on the motion of the Moon around the Earth. ${ }^{26}$ But he did not explain his curvature approach in the first edition of his Principia of 1687; it appeared as an alternate method for solving this problem only in the second and third editions of 1713 and 1726 . As a consequence, his early curvature approach has been regarded erroneously as his more mature development of orbital dynamics. ${ }^{27}$

In Newton's curvature approach, Kepler's area law (or conservation of angular momentum) had remained hidden; ${ }^{28}$ he discovered its physical origin - that the acting

* James Crompton (1648-1694), an astronomer who was elected as a Fellow of Jesus College, Cambridge, in 1672 . 


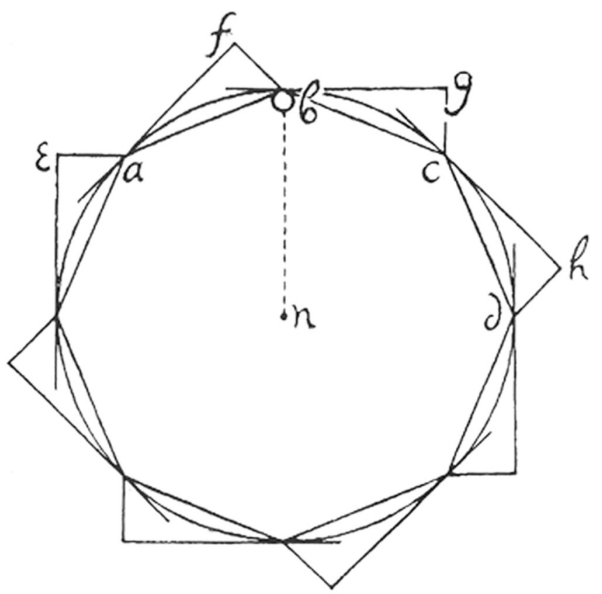

Fig. 3. Newton's impulse diagram for circular motion, showing his polygonal approximation of circular motion for a body under the action of a series of periodic impulses directed toward a fixed center at $n$. Each tangential displacement $a f, b g$, and $c h$ between impulses of length $v \delta t$, where $v$ is the initial velocity and $\delta t$ is the time bewteen impulses, is compounded with a corresponding perpendicular displacements $f b, g c$, and $h d$ due to the impulses, to obtain a polygonal trajectory $a b c d . .$. with vertices on a circle with center at $n$ and radius $b n$. It is then straightforward to show that in the limit of vanishing $\delta t$ the acceleration toward the center $\mathrm{n}$ is $2 f b /(\delta t)^{2}$, where $v=a b / \delta t$ is the velocity of the body and $r=$ $b n$ is the radius of the circular orbit. Source: Whiteside, "Prehistory" (ref. 17), p. 13.

force must be directed toward a fixed center - only after implementing Hooke's way of "compounding" velocities geometrically for a general orbit. ${ }^{29}$ Newton had considered an approach similar to Hooke's in his earliest work on dynamics, as shown in his "Waste Book" of 1664 (figure 3), ${ }^{30}$ but he evidently did not consider extending it to arbitrary motion until after Hooke prompted him to do so. Kepler's area law was crucial to Newton's development of orbital dynamics, because it permitted him to express the time variable in purely geometrical terms, since "The areas which bodies [planets] made to move in orbits describe by radii drawn to an unmoving center of force lie in unmoving planes and are proportional to the times." 31 Newton's proof of Kepler's area law is one of the cornerstones of his Principia, which he presented in Book 1, Proposition 1 , Theorem I.

\section{Hooke's Formulation of the Principles of Orbital Dynamics}

Hooke's profound physical intuition, which was guided by his numerous experiments (thought to be on the order of several hundred), led him during the middle 1660s to a correct qualitative formulation of the principles of dynamics as applied to celestial mechanics. He stated that the orbital motion of a planet is determined by "compounding" its inertial motion along a straight line with an attractive motion toward the Sun impressed by the gravitational attraction of the Sun. Furthermore, in 1674 he formu- 
lated a concept of universal gravitational attraction, ${ }^{33}$ and he later deduced the inverse-square law based on his conjecture that gravity originates in periodic pulses from matter, in analogy to the emission of light and sound. ${ }^{34}$ While crediting Hooke with some of these ideas and acknowledging his influence on Newton, historians of science generally have concluded, with few exceptions, ${ }^{35}$ that Hooke was unable to obtain a quantitative or mathematical formulation of his principles of dynamics.

This judgment echoes the charges that Newton leveled against Hooke in a letter to Halley of June 20,1686, that:

Borel* did something in it \& wrote modestly, he [Hooke] has done nothing \& yet written in such a way as if he knew \& had sufficiently hinted all but what remained to be determined by ye drudgery of calculations \& observations, excusing himself from that labour by reason of his other business: whereas he should rather have excused himself by reason of his inability. For tis plain by his words he knew not how to go about it. Now is not this very fine? Mathematicians that find out, settle \& do all the business must content themselves with being nothing but dry calculators $\&$ drudges $\&$ another that does nothing but pretend \& grasp at all things must carry away all the invention as well of those that were to follow him as of those that went before.... For as Borell wrote long before him that by a tendency of ye Planets towards ye sun like that of gravity or magnetism the Planets would move in Ellipses, so Bullialdus** wrote that all force respecting ye Sun as its center \& depending on matter must be reciprocally in a duplicate ratio of ye distance from ye center.... ${ }^{36}$

Newton's diatribe followed after he had heard that Hooke had claimed that Newton had plagiarized from him the discovery of the inverse-square law of the gravitational force. Halley, in his capacity as editor of the Principia, had tactfully written to Newton on May 22, 1686, that:

Mr Hook has some pretensions upon the invention of ye rule of the decrease of Gravity, being reciprocally as the squares of the distances from the Center. He sais you had the notion from him, though he owns the Demonstration of the Curves generated therby to be wholly your own; how much of this is so, you know best.... ${ }^{37}$

Newton also had criticized Hooke's claim that the inverse-square law implied that the planets move in elliptical orbits around the Sun, pointing out that Hooke had concluded incorrectly that their velocities in such orbits vary inversely with their distances from the Sun. What Newton conveniently forgot in his effort to discredit Hooke is that in Hooke's letter to him in 1679 , Hooke had correctly described some of the principles of orbital motion that led Newton to his discovery of Kepler's area law, and to a deep-

* Giovanni Alfonso Borelli (1608-1679), Italian physicist and astronomer who published Theorica Mediceorum Planetarum ex causis physicis deductee in 1666 in which he supposed that a centrifugal tendency acted on a planet and counterbalanced the gravitational attraction of the Sun.

** Ismael Bouillaud (Ismaelis Bullialdi [Bullialdus],1605-1694), French librarian, priest, and eminent astronomer and mathematician who published Astronomia Philolaica in 1645 based upon Copernicus's and Kepler's discoveries. 
er understanding of orbital dynamics. ${ }^{38}$ Newton later referred to Hooke in his System of the World, the less mathematical treatment of Book III of the Principia, lumping him together with other well-known natural philosophers whose speculations about the motion of the planets were wrong: "The later philosophers pretend [my italics] to account for it either by the action of certain vortices, as Kepler and Descartes; or by some other principle of impulse or attraction, as Borelli, Hooke, and others of our nation." 39

Actually, Hooke arrived at his remarkable physical insights in dynamics by careful observations of mechanical analogs of celestial motion, and not merely by guessing. With the notable exceptions of Johannes Lohne and V.I. Arnol'd, ${ }^{40}$ many Newtonian scholars have repeated the misconception that Hooke's discoveries of the principles of mechanics and the inverse-square law of gravitational force were pure guesses, or somehow based on incorrect mathematical reasoning. A. Rupert Hall, for example, has stated that, "Other philosophers of mechanics, such as Marcus Marci* and Robert Hooke, were just as deeply embroiled in imprecise notions and perilous analogies as was Borelli", ${ }^{41}$ and further, that:

One sees his [Newton's] point: Hooke had been almost as vague as Borelli, and certainly could never have produced dynamical demonstrations applicable to planetary motion: yet we may allow that the idea of a terrestrial projectile becoming a satellite in elliptical orbit was Hooke's own, though a "guess" indeed as Newton rightly called it. ${ }^{42}$

This sentiment, that Hooke did not have any sound basis for his physical principles, echoes the words of the eighteenth-century French mathematician Alexis-Claude Clairaut (1713-1765) who, although considered to be a supporter of Hooke, stated that Hooke's examples "serve to show what a distance there is between a truth that is glimpsed and a truth that is demonstrated." 43

Hooke's dynamical principles, however, were from the outset grounded on careful experiments and observations of mechanical systems that could serve as analogs of celestial dynamics. The best-documented example is that of the motion of a circular or conical pendulum, ${ }^{44}$ but there also is evidence that he studied the dynamics of balls rolling inside various surfaces of revolution. These mechanical systems serve as approximate analog models for different central attractive forces. Indeed, for a long time Hooke had been applying the maxim "That Nature seems to take similar Ways for producing similar Effects; without granting of which we cannot reason or make any Conclusion from similar Operations." ${ }^{45} \mathrm{He}$ cautions, however, that "Omne simile non est idem [Everything that looks the same is not equal]." 46

Patri J. Pugliese has recently reproduced a remarkable diagram (figure 4), ${ }^{47}$ which is part of an unfinished and unpublished manuscript that Hooke wrote, entitled "The

* Marcus Marci (1595-1667), Bohemian physician and professor of medicine at the University of Prague (1620-1660) who published De proportione motus in 1639 describing his theory and experiments on collisions of bodies, and Thaumantias liber de arcu coelesti in 1648 reporting his researches on optics. 


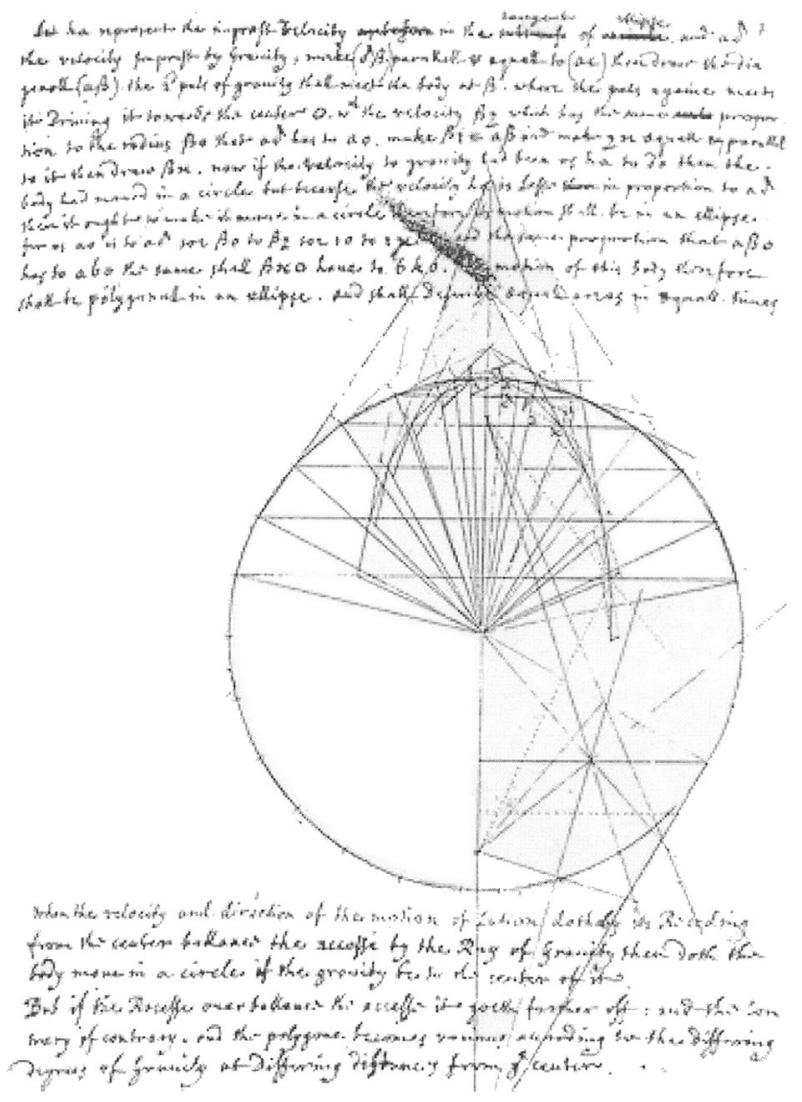

Fig. 4. Hooke's September 1685 geometrical construction and graphical evaluation of the orbital motion of a body in a central field of force that varies linearly with distance. The original is in Hooke's manuscripts in Trinity College Library, Cambridge, Ms O.11a.1/16. Reproduced by permission of the Master and Fellows of Trinity College.

Laws of Circular Motion," which is deposited in the Wren Library, Trinity College, Cambridge. It shows a graphical construction of a segment of an orbit for a body moving in a central field of force, and on a page associated with it is inscribed the date September 1,1685 . This date, as we shall see, is important in relating it to Newton's $D e$ Motu ${ }^{48}$ the preliminary draft of his Principia, which was registered by Halley at the Royal Society in November 1684. Hooke's graphical construction shows a body moving under the action of an attractive force that varies linearly with the distance from the center of its orbit, and in the handwritten text associated with it he states that its orbit is that of an ellipse. Pugliese has analyzed it only for the special case of circular motion and has implied that Hooke failed to generalize his construction to noncircular motion, concluding that "Hooke claims, but certainly does not demonstrate, [its orbit] to be elliptical." Pugliese asserted earlier that, "There can be no doubt that Hooke 
could not have taken this [dynamical] principle so far [as Newton]," and he commented further that Hooke "does not seem to have ever come to a full appreciation of the magnitude of the step from his ideas to Newton's achievement." 49

Pugliese's comments and conclusions thus seem to reinforce the conventional wisdom among historians of science about Hooke's mathematical limitations. I have carried out a detailed analysis of Hooke's diagram, however, which leads to very different conclusions. ${ }^{50}$ Hooke's graphical solution for the orbit of a body moving in a central field of force that varies linearly with the distance from the center of its orbit is based precisely on the same geometrical construction that Newton developed the preceding year in Theorem 1 of his De Motu, and which then became Book I, Proposition I, Theorem I, two years later in his Principia. Hooke's geometrical construction is effectively the mathematical formulation of the principles of dynamics that he had been proposing during the previous twenty years. Moreover, contrary to Pugliese's assertion, I have shown that Hooke in his diagram gives several different graphical demonstrations that the vertices of the resulting polygonal orbit lie on an ellipse, and that this is an exact property of his construction, which is equivalent to an affine transformation of a circle into an ellipse. ${ }^{51}$

While the extent to which members of the Royal Society were aware of Newton's De Motu is uncertain,* at least two letters exist indicating that Hooke appears to have seen it shortly after it was registered at the Royal Society in November 1684. Thus, a month later Flamsteed wrote to Newton that, "I am obliged by your kind concession of ye perusall of your papers, tho I beleive I shall not get a sight of them till our common freind [friend] Mr Hooke \& the rest of the towne have beene first satisfied." ${ }^{52}$ And on June 29, 1686, Halley wrote to Newton that "it [De Motu] has been enterd upon the Register books of the Society as all this past Mr Hook was acquainted with it...." 53

Assuming that Hooke saw and read Newton's De Motu, he would have recognized that Newton had implemented geometrically his dynamical principle of compounding a tangential velocity with an impressed radial acceleration due to a center of attraction.** Thus, Newton had drawn a diagram on the first page of his De Motu (figure 5) that describes a geometrical construction that embodies Hooke's dynamical principles for a body undergoing a sequence of impulses under the action of a central force. In it,

* Pugliese believes that Newton's De Motu was not "generally known to members of the Royal Society prior to the appearance of his Principia," the "principal argument" being "the total lack of discussion of its contents"; see Pugliese, "Hooke" (ref. 16), p. 203. Likewise, Mordechai Feingold, who is familiar with the history of the Royal Society, also believes that Halley did not circulate Newton's De Motu to members of the Royal Society; private communication, January 9, 2004.

** When the Principia was published in 1687, most of its readers, among whom were some of the best mathematicians in Europe, had considerable difficulties in understanding its contents and in particular the significance of Kepler's area law. For example, the French mathematician Pierre Varignon (1654-1722) assumed different laws for the time evolution of orbital motion, such as those proposed by the English astronomer Seth Ward (1617-1689) and the Italian-French astronomer Gian Domenico Cassini (1625-1712); see Guicciardini, Reading the Principia (ref. 1), pp. 202-205. Therefore it is revealing that Hooke recognized that Newton's proof of Kepler's area law in his De Motu was based upon his own principles of planetary motion. 


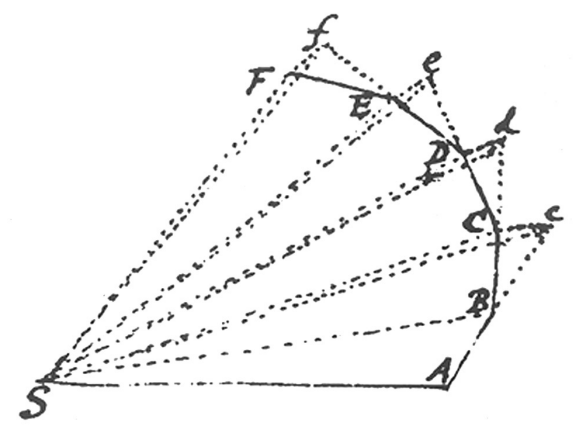

Fig. 5. Diagram associated with Newton's proof of Kepler's area law as it first appeared in his De Motu of 1684. Source: Whiteside, "preliminary manuscripts" (ref. 15), p. 3.

the trajectory $A B C D E F$ is determined by the initial tangential velocity described by the length $A B$ and by the impressed deflections $c C, d D, e E$, and $f F$ that are directed toward the center of force at $S$, or as Newton wrote:

Let the time be divided into equal intervals, and in the first interval of time suppose the body by reason of its innate force describes the line $A B$. Likewise in the second interval of time if nothing were to impede it suppose it would continue straight on to $c$ covering a length $B c$ equal to the line $A B$, so that the radii $A S, B S, c S$ being drawn to the center [ $S$ ] the areas $A S B, B S c$ would be made equal. But actually when the body comes to $B$ let the centripetal force act [on it] with a single great impulse, forcing the body to deviate from the line $B c$ and continue on in the line $B C .^{54}$

Newton applied this geometrical construction to prove Kepler's area law, that "All bodies circulating about a centre [of force] sweep out areas proportional to the times." 55

Whether or not Hooke had seen Newton's De Motu, he gave his own description of the geometrical construction in his diagram of 1685 (figure 4) in the handwritten text above it in the same physical terms that he had used for the past twenty years:

Let $h a$ represent the imprest velocity in the tangent of an ellipse and $a \delta$ the velocity imprest by Gravity. Make $(\delta \beta)$ parallel and equal to $(a c)$, then draw the diagonall $(a \beta)$. The second puls of gravity [referring to Hooke's theory of periodic gravitational pulses] shall meet the body at $\beta$ where the puls againe meets it, driving it towards the center $o$ with the velocity $\beta \gamma$ which has the same proportion to the radius $\beta o$ that $\alpha \delta$ has to $a o .56$

Hooke's description is similar to, but not merely a translation of Newton's description from Latin into English, quoted above. Furthermore, Hooke proceeds in his geometrical construction in a way quite different from Newton by applying it in a novel way to obtain the orbit of a body moving in an attractive central field of force that varies linearly with distance. We can see this more clearly by deleting some of Hooke's auxiliary lines and enlarging the result, as shown in figure 6. We see that Hooke's diagram cor- 


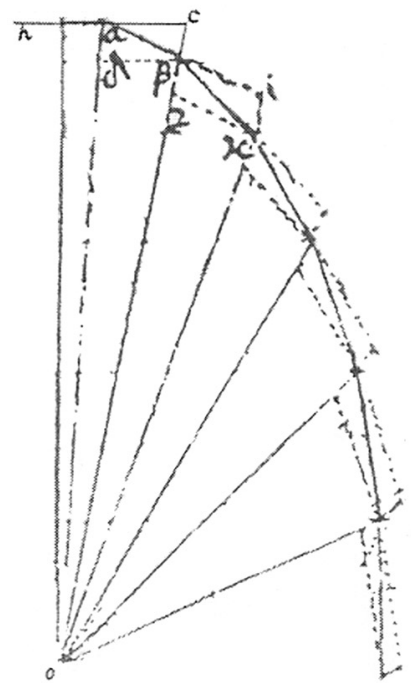

Fig. 6. Enlargement of the upper-right-hand portion of Hooke's diagram of September 1685 (figure 4) with some auxiliary lines removed to show more clearly its correspondence to Newton's diagram in his De Motu of 1684 (figure 5). Reproduced by permission of the Master and Fellows of Trinity College.

responds to Newton's, taking into account that Hooke's proceeds clockwise with the initial velocity of the body directed along $h a$, while Newton's proceeds counterclockwise with its initial velocity directed along $A B$.

\section{Background to Hooke's Physical Principles of Orbital Motion}

I now turn to how Hooke developed his understanding of orbital dynamics and discuss further aspects of his correspondence with Newton in 1679, as well as Halley's and Newton's correspondence in 1686.

Hooke gave a remarkable lecture to the members of the newly founded Royal Society on May 23, 1666, entitled "A Statement of Planetary Movements as a Mechanical Problem," 57 in which he proposed that the Keplerian elliptical orbits of planets around the Sun could be obtained by compounding an inertial straight-line motion with an inflection toward the center of the Sun due to an attractive property of the Sun. This was the first published account in which some of the essential dynamical principles of planetary motion were stated clearly and unambiguously, nearly two decades before Newton implemented them in mathematical form in his celebrated Principia. Hooke's paper, which was registered by the Royal Society, begins with his statement that:

I have often wondered, why the planets should move about the sun according to Copernicus's supposition, being not included in any solid orbs (which the ancients possibly for this reason might embrace) nor tied to it, as their centre, by any visible strings; and neither depart from it beyond such a degree, nor yet move in a straight line, as all bodies, that have one single impulse, ought to do.... 58 
Hooke then dismisses a theory, partly due to Borelli, ${ }^{59}$ which was in vogue at the time, that the impressed force is due to a medium of variable density acting on the planetary body, and states his own idea that:

the second cause of inflecting a direct motion into a curve may be from an attractive body placed in the centre; whereby it continually endeavours to attract or draw it to itself. For if such a principle be supposed, all the phenomena of the planets seem possible to be explained by the common principle of mechanic motions; and possibly the prosecuting [of] this speculation may give us a true hypothesis of their motion, and from some few observations, their motions may be so far brought to a certainty, that we may be able to calculate them to the greatest exactness and certainty that can be desired.

This inflexion of a direct motion into a curve by a supervening attractive principle I shall endeavour to explicate from some experiments with a pendulous body: not that I suppose the attraction of the sun to be exactly according to the same degrees, as they are in a pendulum.... ${ }^{60}$

Hooke thus proposed that a conical pendulum, by projecting its motion onto a plane perpendicular to its axis of suspension, could serve as a mechanical analog to demonstrate the principles of planetary orbital motion. His analog model therefore generalized Descartes's demonstration of circular motion of a stone revolving on a sling (figure 1). Hooke discussed the theory of the conical pendulum, pointing out that the effective radial force on its bob

is greater and greater, according as it is farther and farther removed from the centre, which seems to be otherwise in the attraction of the sun....

But however it be, the compounding this motion with a direct or straight motion just crossing it, may serve to explicate this hypothesis, though all the appearances of it are not exactly the same. ${ }^{61}$

Hooke thus was well aware of the shortcomings of the conical pendulum as a model for planetary orbital motion.

Hooke was then Curator of the newly founded Royal Society, and one of his main tasks was to present weekly scientific experiments. He gave a demonstration to its members of "a pendulum fastened to the roof of the room with a large wooden ball of lignum vitae on the end of it." 62 This would have given an impressive demonstration of nearly closed elliptical orbits as projected onto a plane normal to its axis of suspension, as I verified by repeating Hooke's experiment. I suspended a weight from the ceiling of a room, attached a pen to it, and traced its motion on a sheet of paper on the floor, as shown in figure $7,{ }^{*}$ which also confirmed Hooke's careful observation that "the progression of the auges [apsides] are very evident." 63

* Richard S. Westfall commented in a letter to me of January 22, 1995, that "your tracing of the ellipse is indeed quite remarkable. I have been willing, apriori, to assert with confidence that anything half so precise was impossible." His remark underscores the importance of repeating often-neglected historical experiments to understand their significance in shaping theoretical concepts. 


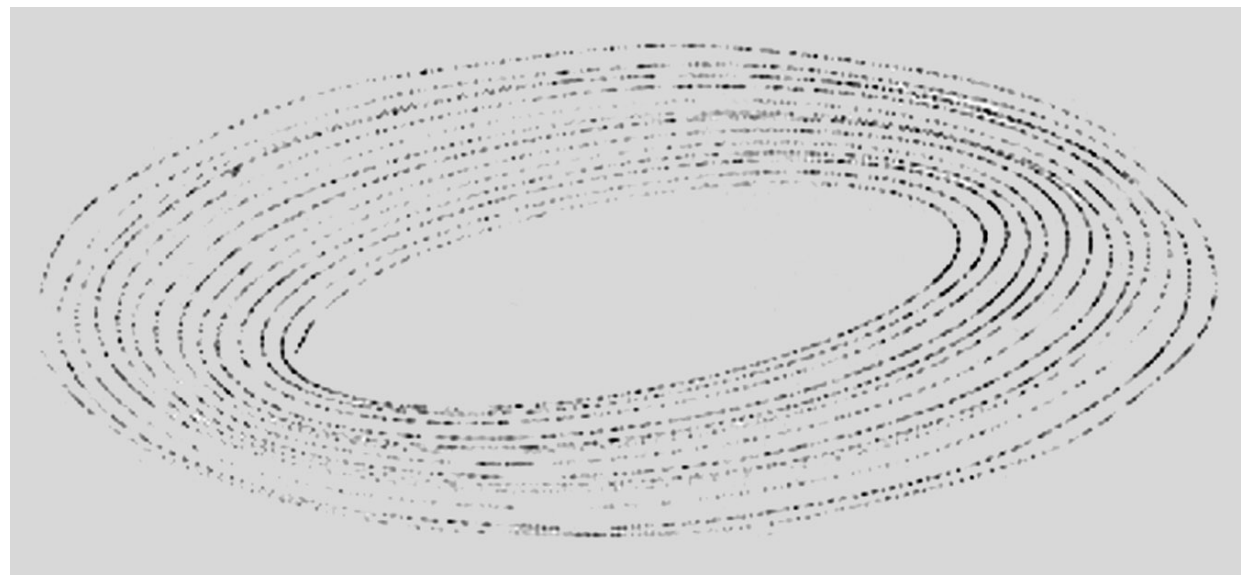

Fig. 7. The trajectory of a conical pendulum as determined experimentally by the author.

Later, in a letter that Hooke wrote to Newton on December $6,1679,{ }^{64}$ he made a drawing (figure 8) of a similar orbit of a body revolving around the center of the Earth "supposing then ye earth were cast into two half globes," which clearly shows a "progression of the auges." * Nevertheless, in the vast literature on the Newton-Hooke correspondence, Hooke's diagram has invariably been reproduced incorrectly as a symmetrical figure ${ }^{65}$ which leaves out the "progression of the auges." Hooke, moreover, demonstrated mathematically that in this case the horizontal force on the bob increases linearly with the distance from its axis of suspension. ${ }^{66}$

Extending the pendulum's axis of suspension to a point on the plane normal to it gives a point that coincides with the center of its elliptical motion, while the center of force for the motion of a planet is at a focal point of its elliptical orbit. Furthermore, the period of the pendulum is nearly independent of the size of its orbit, as Hooke discovered for the oscillation of springs.** This is in striking contrast to the dependence of the periods of planets on their distances from the Sun, which obey Kepler's third law, that is, the square of their periods vary with the cube of the major axes of their elliptical orbits. But, as noted above, Hooke was well aware of the shortcoming of his model for planetary motion. He also attached a smaller pendulum to it to model, although less

* The precession of the apsides (auges) arises because for noncircular motion the trajectory of a conical pendulum does not lie in a plane. The precession can be readily calculated for small eccentricities of the orbit.

** In his sixth Cutler Lecture, which he published in 1678, Hooke deciphered his anagram, ceiiinosssttuu, presented two years earlier, which translates to ut tensio sic vis (as the extension so the force); see Hooke, "De Potentia Restitutiva" (ref. 9), pp. 333-334. It is not generally recognized that Hooke was able to obtain the correct phase-space relation between velocity and position for the harmonic oscillator, shown in a graph in a beautiful frontispiece of his paper. $\mathrm{He}$ also showed that the period of the spring was independent of amplitude, but he did not obtain the correct dependence of amplitude and velocity on time. 


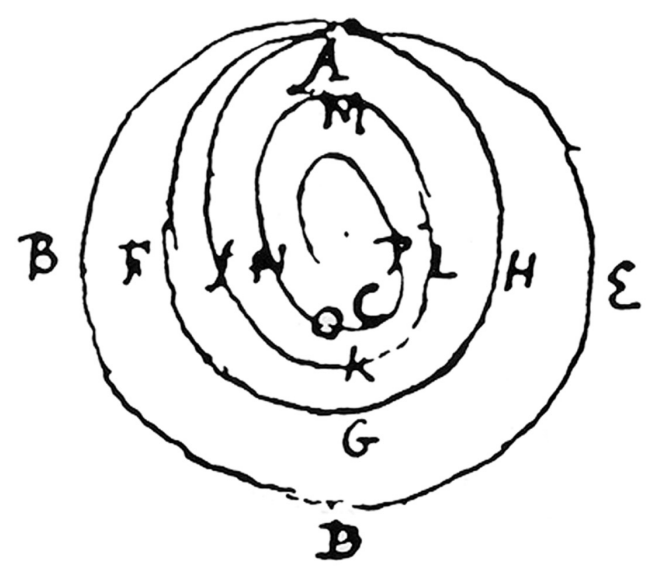

Fig. 8. Hooke's drawing for the trajectory of a body moving inside the Earth, supposing that the Earth were divided into two half globes. Source: Koyré, "Unpublished Letter" (ref. 6), p. 330.

successfully, the motion of the Moon around the Earth. This is particularly significant, because it shows that Hooke understood the universal character of the gravitational force, which he later enunciated explicitly. He also proposed and carried out several other inconclusive experiments to determine how the gravitational force varies with the distance both above and below the surface of the Earth. ${ }^{67}$

Hooke developed his ideas further in his Cutler Lectures, which he delivered at the regular meetings of the Royal Society. His first lecture, which he delivered in 1670, was on An Attempt to Prove the Motion of the Earth from Observations by observing stellar parallax* and thus to "furnish the Learned with an experimentum crucis to determine between the Tychonick and the Copernican Hypotheses." 68 Two years earlier, in October $1668,{ }^{69}$ he had begun to erect a zenith telescope in his quarters in Gresham College in London (figure 9), and in 1670 he began to make observations of the star Gamma Draconis as it passed directly overhead.** Now, at the end of his lecture in 1670 , which he did not publish until $1674,{ }^{70}$ he restated his principles of dynamics and formulated the principle of universal gravitational attraction:

* The idea to demonstrate the motion of the earth by observing stellar parallax can be traced back to the ancient Greeks.

** After a few months, Hooke observed a displacement in the expected position of Gamma Draconis, but he tells us that unfortunately the objective lens of his telescope accidentally broke and he therefore discontinued his observations; see his Attempt (ref. 33), p. 24. The English astronomers James Bradley (1693-1762) and Samuel Molyneux (1689-1728) repeated Hooke's observations in 1725 with a better telescope and discovered that the observed displacements could not be due to parallax, but they were unable to interpret them. By 1727, however, after erecting another telescope and continuing his observations, Bradley found the explanation as due to the finite velocity of light, which the Danish astronomer Ole Römer (1644-1710) had determined in 1676. Bradley thus was able to establish the motion of the Earth, finally accomplishing Hooke's Attempt to Prove the Motion of the Earth from Observations. See "A Letter 
At which time also I shall explain a System of the World differing in many particulars from any yet known, answering in all things to the common Rules of Mechanical Motions: This depends on three Suppositions. First, That all Cœlestial Bodies whatsoever, have an attraction or gravitating power towards their own Centers, whereby they attract not only their own parts, and keep them from flying from them, as we may observe the Earth to do, but that they do also attract all the other Cœlestial Bodies that are within the sphere of their activity; and consequently that not only the Sun and Moon have an influence upon the body and motion of the Earth, and the Earth upon them, but that [Mercury] also [Venus], [Mars], [Saturn], and [Jupiter] by their attractive powers, ${ }^{*}$ have a considerable influence upon its motion as in the same manner the corresponding attractive power of the Earth hath a considerable influence upon every one of their motions also. The second supposition is this, That all bodies whatsoever that are put into a direct and simple motion, will so continue to move forward in a streight line, till they are by some other effectual powers deflected and bent into a Motion, describing a Circle, Ellipsis, or some other more compounded Curve Line. The third supposition is, That these attractive powers are so much the more powerful in operating by how much the nearer the body wrought upon is to their own Centers. Now what these several degrees are I have not yet experimentally verified.... ${ }^{71}$

In light of Newton's later unkind description of Hooke, as quoted in my introduction, we must remember that Hooke was a poorly paid employee of the Royal Society, whose aristocratic members often ordered him to carry out this or that demonstration almost every week during the year. ${ }^{72} \mathrm{He}$ did not have the advantage of inherited wealth like his early mentor, Robert Boyle (1627-1691), or that of a well-paid academic chair like Newton, which would have given him the leisure to follow his own intellectual pursuits. Instead, Hooke supplemented his income by moonlighting, for example, by serving as one of the main surveyors for the reconstruction of the City of London after the Great Fire in $1666,{ }^{73}$ by designing buildings and private houses, and by attempting to patent his inventions.

Hooke discussed his ideas with Newton in letters to him in 1679. By then Hooke had become Secretary of the Royal Society, and his avowed purpose in his first letter was to again establish contact with Newton, with whom he had a strained relationship since their earlier controversy on optics, and to elicit Newton's reaction to his current physical hypotheses. Thus, he wrote to Newton on November 24, 1679, that:

For my own part I shall take it as a great favour if you shall please to communicate by Letter your objections against any hypothesis or opinion of mine, And particu-

from the Reverend Mr. James Bradley Savilian Professor of Astronomy at Oxford, and F.R.S. to Dr. Edmund Halley, Astronom. Reg. \&c. giving an Account of a new discovered Motion of the Fix'd Stars," Philosophical Transactions 35 (1727), 637-661; reprinted in Miscellaneous Works and Correspondence of the Rev. James Bradley, D.D. F.R.S. (Oxford: Oxford University Press, 1832; reprinted New York and London: Johnson Reprint Corporation, 1972), pp. 1-16.

* I have substituted the names of the planets for the accepted symbols for them that Hooke used. 


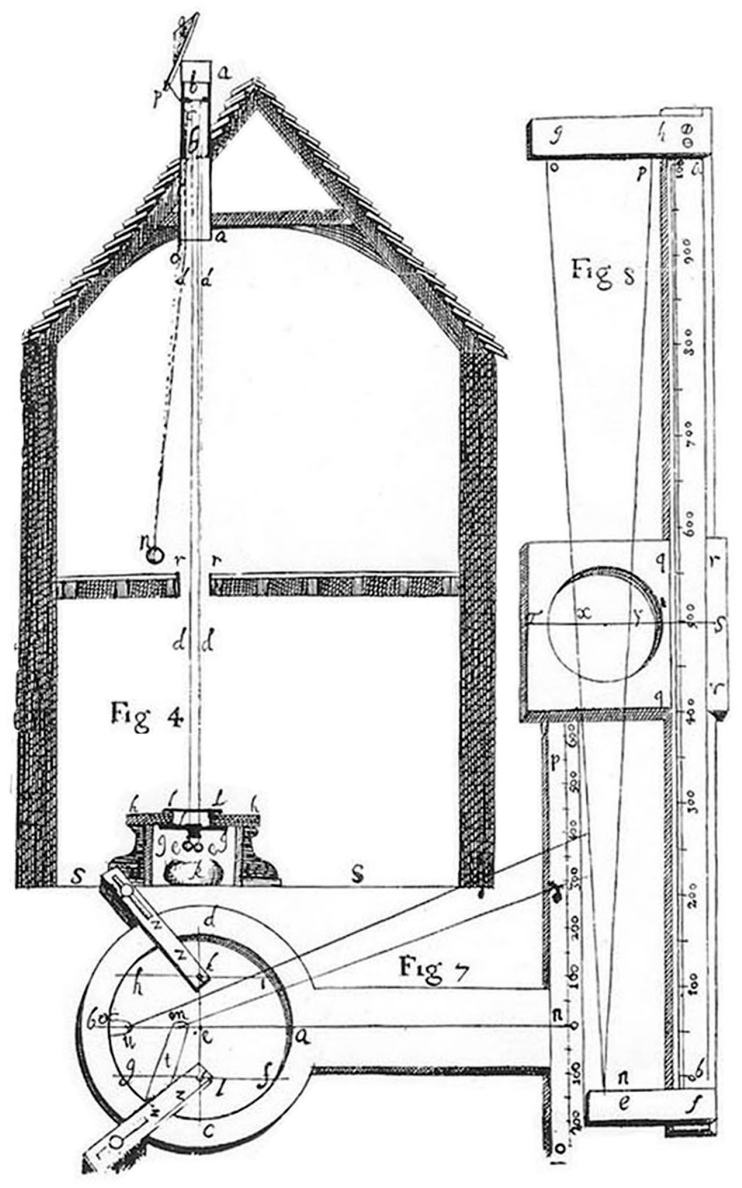

Fig. 9. Hooke's zenith telescope, which he designed to follow the star Gamma Draconis as it passed directly above his lodgings in Gresham College, is shown in his Fig. 4. He inserted a wooden tube 1 foot square and about 10 feet long through a hole in the roof and cemented the objective lens $c$ of his telescope (focal length about 36 feet) inside it, which he protected against rain by closing the lid with a cord. He put the eyepiece in a hole in the table at the bottom, above which he placed his measuring instrument, the round thin brass plate shown on the left of his Fig. 7, insuring that it lay perpendicular to the optical axis of his telescope by using the plumb lines with the small lead balls on their lower ends. The cross hairs $a b$ and $c d$, which lay exactly east-west and north-south, crossed at $e$, so that as a star passed through the zenith its position on the meridian was determined by the distance em, whose observed magnitude was magnified 15 times in his eyepiece, and which he measured to seconds of arc with the ingenious micrometer he invented shown in his Fig. 8. He took observations by lying on the couch $k$ underneath the table in his Fig. 4, looked directly upward through the eyepiece, and observed accurately how far north-south from the zenith $e$ the star passed the meridian $d c$ while an assistant recorded its time of passage using a pendulum clock and its distance using the micrometer scale op. Hooke observed the star Gamma Draconis four times between July 6 and October 20, 1669, concluding that he had found a "sensible parallax" owing to the motion of the Earth about the Sun. Source: Gunther, Early Science. Vol. VIII. Cutler Lectures (ref. 3), plate after p. vii. 
larly if you will let me know your thoughts of that of compounding the celestiall motions of the planetts of a direct motion by the tangent \& an attractive motion towards the centrall body. ${ }^{74}$

It thus appears from this letter and from his later correspondence with Newton that one of Hooke's principal reasons for now writing to Newton was that he had been unable to make progress in expressing his physical principles of celestial mechanics mathematically, and he wanted to solicit help from Newton, whose great mathematical abilities were known to several members of the Royal Society. Hooke's diary shows that he had approached other mathematicians earlier for help without success. He now also discussed with Newton the motion of a body inside the Earth, which was mostly of concern to him insofar as it clarified the orbital dynamics for central forces, which he had been studying with mechanical analogs for the past fourteen years. Thus, Newton responded on December 13, 1679, sending Hooke a drawing of the orbit of a body moving under the action of a constant radial force (figure 2$),{ }^{75}$ to which Hooke replied on January 6,1680 , that:

Your Calculation of the Curve by a body attracted by an æquall power at all Distances from the center Such as that of a ball Rouling in an inverted Concave Cone is right and the two auges [apsides] will not unite by about a third of a Revolution. ${ }^{76}$

Newton's letter of December 13 must have come as a great revelation to Hooke. In his response to it quoted above, Hooke indicated that he had previously observed the orbit of a ball rolling inside an inverted cone, and that he knew that the effective radial force acting on it is constant. He also realized that Newton had developed a method for calculating such an orbit. But almost all historians of science have ignored Hooke's insightful observations.*

I repeated Hooke's experiment of a ball rolling inside an inverted cone and took a stroboscopic picture of its trajectory (figure 10), which evidently corresponds approximately to the theoretical trajectory Newton drew (figure 2), although the angle between successive apsides is somewhat different. Hooke continued:

But my supposition is that the Attraction always is in a duplicate proportion to the Distance from the Center Reciprocall, and Consequently that the Velocity will be in a subduplicate proportion to the Attraction and Consequently as Kepler Supposes Reciprocall to the Distance. ${ }^{77}$

* D.T. Whiteside quoted Hooke without however indicating that Hooke was referring to an experimental observation relevant to Newton's diagram, remarking only that:"Inadequate or no, even such relatively unsophisticated reasonings [Newton's] were above Hooke's head and he could only compliantly answer..."; see Whiteside, ed., The Mathematical Papers of Isaac Newton. Vol. VI. 1684-1691 (Cambridge: Cambridge University Press, 1974), p. 12. I have shown, however, that Newton's reasonings were far from unsophisticated and instead demonstrated a remarkable theoretical understanding of the motion of a body moving under the action of a constant attractive central force, in good agreement with Hooke's observations; see Nauenberg, "Newton's Early Computational Method" (ref. 15). 


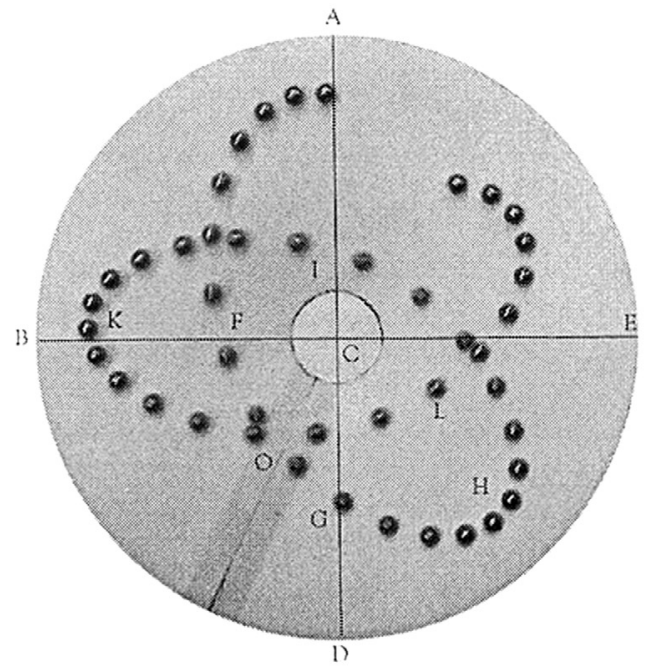

Fig. 10. Stroboscopic photograph taken by the author of the trajectory of a ball revolving inside an inverted cone. The angle between successive "auges" (apsides) depends on the cone's angle of aperture. For the ball to move sufficiently slowly to determine the shape of the orbit with the naked eye, I chose an angle of aperture of 60 degrees, which also brings the trajectory of the ball in closer agreement with Newton's diagram (figure 2) and compensates for the error in his drawing. By a historical quirk, it appears that Hooke used a similar angle of aperture.

Here Hooke announced for the first time his belief that the gravitational force depends inversely on the square of the distance of a planet from the Sun. He erred, however, in saying that Kepler took its velocity to depend inversely on the radial distance. Newton later pounced on Hooke's error, claiming that Hooke did not understand orbital motion under a central inverse-square force, but ignoring that Hooke in his letter went further and supposed correctly:

that with Such an [inverse-square] attraction the auges [apsides] will unite in the same part of the Circle and that the neerest point of accesse to the center will be opposite to the furthest Distant. Which I conceive doth very Intelligibly and truly make out all the Appearances of the Heavens. ${ }^{78}$

Nowhere, however, is there any evidence in Hooke's letter that he knew at this time how to demonstrate his conjecture mathematically. Instead, he goes on, stating that:

(though in truth I agree with You that the Explicating the Curve in which a body Descending to the Center of the Earth, would circumgyrate were a Speculation of noe Use yet) the finding out the proprietys of a Curve made by two such principles will be of great Concerne to Mankind.... This Curve truly Calculated will shew the error of those many lame shifts [ad hoc approximations] made use of by astronomers to approach the true motion of the planets with their tables. ${ }^{79}$ 
Finally, Hooke states again in a letter to Newton on January 17, 1680, that "I doubt not but that by your excellent method you will easily find out what that Curve must be, and its proprietys, and suggest a physicall Reason of this proportion." 80

Hooke's remarks indicate that at this time he did not know how to calculate the orbital motion of a planet in an attractive central field of force; his remarkable physical understanding and hypothesis were based on mechanical analog models. However, his asking Newton for mathematical help turned out to be a capital mistake. Newton formulated Hooke's problem mathematically and solved it for various central forces, including the inverse-square force, but he never acknowledged Hooke's seminal physical concept or even replied to Hooke's letter of January 17. Two months earlier, however, on November 28, 1679, Newton had written to Hooke that:

I did not before ye receipt of your last letter [sent four days earlier], so much as heare (yt [that] I remember) of your Hypotheses of compounding ye celestial motion of ye Planets, of a direct motion by the tang[en]t to ye curve....

If I were not so unhappy as to be unacquainted with your Hypotheses abovementioned.... ${ }^{81}$

Newton went on to congratulate Hooke "that so considerable a discovery as you made of ye earth's annual parallax is seconded by Mr. Flamstead's Observations." ${ }^{82}$ Hooke, however, had not told Newton anything in his last letter about his own apparent discovery of the Earth's parallax, but only had commented briefly that Flamsteed "hath confirmed the paralax of the orb of the earth." 83

It thus appears that Newton was familiar with Hooke's tract on An Attempt to Prove the Motion of Earth by Observations, ${ }^{84}$ as Newton later admitted to Halley in a letter of July $27,1686 .{ }^{85}$ Hooke himself was not convinced of Newton's denials and later wrote in an unpublished memorandum that, "Newton pretends he knew not Hooke's Hypoth. as by his Answer to ye former dated Nov. $28,1679 . . . .86$ Hooke also scribbled this telling comment on the margin of Newton's letter to him of that date.

Four years earlier, in 1674, Hooke had published his hypotheses ${ }^{87}$ which evidently were read by some of the leading scientists at the time, such as Christiaan Huygens (1629-1695) and Gian Domenico Cassini (1625-1712), Director of the Paris Observatory, who had sent comments on them to the Philosophical Transactions. ${ }^{88}$ Also, as noted above, Hooke communicated his ideas directly to Newton in 1679. A decade later, on February 15, 1689, Hooke noted in his diary that, "At Hallys [Halley's] met Newton; vainly pretended claim yet acknowledged my information. Interest has no conscience: A posse ad esse non valet consequentia [It is not valid to infer from the possible to the actual]." 89

Hooke had mentioned to Newton in a letter of January 6,1680, without giving any supporting arguments, that:

my supposition is that the Attraction always is in a duplicate proportion to the Distance from the center Reciprocall... [It] truly makes out all the Appearances of the Heavens ... not that I believe there really is such an attraction to the very Center of the Earth.... ${ }^{90}$

In fact, Hooke had conjectured correctly on physical grounds that: 
I rather Conceive that the more the body approaches the Center, the lesse will it be Urged by the attraction - possibly somewhat like the Gravitation on a pendulum or a body moved in a Concave Sphære where the power Continually Decreases the neerer the body inclines to a horizontall motion.... ${ }^{91}$

Newton later also conveniently forgot these remarks and charged incorrectly that "what he [Hooke] told me of ye duplicate proportion was erroneous, namely that it reacht down from hence to ye center of ye earth." 92

Nevertheless, in letters that Newton wrote to Halley in the summer of 1686 that were intended primarily to deny Hooke any credit for the fundamental ideas in his Principia, ${ }^{93}$ Newton admitted that Hooke's letters of 1679 had stimulated him to consider again the fundamental problems of celestial mechanics. Newton had engaged in a bitter invective against Hooke after he had heard rumors that Hooke had accused him of plagiarism, and after he had calmed down following a soothing letter from Halley, ${ }^{94}$ he responded to Halley on July 14,1686 , that:

This is true, that his [Hooke's] Letters occasioned my finding the method of determining Figures, wch when I had tried in ye Ellipsis, I threw the calculation by being upon other studies \& so it rested for about 5 yeares till upon your request I sought for yt [that] paper, \& not finding it did it again \& reduced it into ye Propositions shewed you by Mr. Paget*.... ${ }^{95}$

Two weeks later, on July 27, 1686, Newton again wrote to Halley:

And thô his correcting my Spiral occasioned my finding ye Theorem by wch I afterward examined ye Ellipsis; yet I am not beholden to him for any light into yt [that] business but only for ye diversion he gave me from my other studies to think on these things \& for his dogmaticalnes in writing as if he had found ye motion in ye Ellipsis, wch inclined me to try it after I saw by what method it was to be done. ${ }^{96}$

Seven years earlier, in his letter to Hooke of December 13, 1679, Newton had concluded with the comment that:

Your acute Letter having put me upon considering thus far ye species of this curve, I might add something about its description by points quam proximè. But the thing being of no great moment I rather beg your pardon for having troubled you thus far with this second scribble.... ${ }^{97}$

Newton's computational method "by points quam proxime" has long been puzzling, but I have shown that it was based on his development of the calculus of curvature between 1664 and $1671 .^{98}$ The earliest extant documentary evidence that Newton applied to orbital motion Hooke's idea of compounding the tangential velocity of a body with its radial velocity as impressed by an attractive central force - an idea that Newton claimed repeatedly that he could not remember having heard from Hooke is in Newton's De Motu of 1684, five years after his correspondence with Hooke. This

* Edward Paget (1656-1703?), Fellow of Trinity College, Cambridge, who was appointed as Mathematical Master at Christ's Hospital and was elected as a Fellow of the Royal Society in 1682. 
supports my contention that Hooke had contributed in a fundamental way to Newton's understanding of the dynamical principles that he incorporated in his Principia. Newton later recalled that:

In the end of the year 1679 in answer to a Letter from Dr Hook ... I found now that whatsoever was the law of the forces $w^{\text {ch }}$ kept the Planets in their Orbs, the areas described by a Radius drawn from then to the Sun would be proportional to the times in $\mathrm{w}^{\text {ch }}$ they were described. And by the help of these two Propositions I found that their Orbs would be such Ellipses as Kepler had described. 99

In 1684 Hooke declared to Christopher Wren (1632-1723) and Edmond Halley, two of his friends at the Royal Society, that on the principle of an inverse-square gravitational force "all the Laws of celestiall motions were to be demonstrated, and that he himself had done it....." 100 However, despite a subsequent challenge by Wren, who offered a prize of a 40-shilling book for such a demonstration, Hooke apparently failed to produce a calculation for such planetary motion. ${ }^{101}$ Unfortunately, Hooke also never published his physical arguments for planetary motion or his remarkable graphical solution of 1685 (figure 4) for the motion of a body under the action of an attractive radial force that varies linearly with distance.

\section{Summary and Conclusions}

Hooke's fundamental idea was to determine planetary motion by compounding the planet's tangential velocity with a change in its radial velocity impressed by the gravitational attractive force of the Sun. He gained his profound physical understanding of orbital dynamics by observing mechanical analogs like the motion of a conical pendulum and the motion of a ball rolling inside various surfaces of revolution. I verified Hooke's observations by repeating two of his experiments, one that showed the projection of the trajectory of a conical pendulum onto a plane perpendicular to its axis of symmetry (figure 7), and one that showed the projection of the trajectory of a ball revolving inside an inverted cone (figure 10). Both showed that Hooke's descriptions of these trajectories were fairly accurate.

Hooke also analyzed mathematically the radial forces that act on these bodies toward the axis of symmetry of their motions. ${ }^{102}$ My analysis of Hooke's diagram of 1685 (figure 4), which gave a geometrical construction and graphical evaluation of the path of a body in an attractive radial field of force that depends linearly on the distance, offers new evidence that Hooke came much closer to formulating his principles of dynamics mathematically than previously has been thought. ${ }^{103}$ Hooke developed these principles correctly in the middle 1660 s, demonstrating them with mechanical analogs, and not simply by guessing them as historians of science have assumed. Indeed, he applied precisely the same rules of reasoning that Newton later included in Book III of his Principia. However, prior to the recent publication of Hooke's diagram of 1685 , there was no concrete evidence of the extent to which Hooke had been able to formulate his dynamical principles in mathematical form and apply them to the approximate evaluation of the orbital motion of a body in an attractive central field of force. Hooke apparently was able to accomplish this only after he had seen Newton's 
De Motu of 1684, in which Newton gave an equivalent geometrical construction (figure 3). Newton applied his construction only to obtain a proof of Kepler's area law, however, while Hooke applied his to determine graphically that the orbit of a body moving under the action of an attractive central force that varies linearly with the distance is an ellipse.

The key to a mathematical formulation of the physical principles of orbital motion, which had long eluded Hooke, is to start with the approximation that the central force on a body acts in instantaneous periodic impulses instead of continuously. Hooke actually conjectured that the gravitational attraction originates in periodic pulses, in analogy to the emission of light and sound, ${ }^{104}$ and he deduced from this idea that the strength of gravity varies inversely with the square of the distance from the center of attraction. Newton's letter of December 13, 1679, indicated to Hooke that Newton was capable of evaluating, at least approximately, the orbital motion of a body in an attractive central field of force. In the absence of the infinitesimal calculus that Newton and Leibniz developed, Hooke could have solved the problem of orbital dynamics by his discrete graphical method only approximately.

Among Hooke's unpublished manuscripts in the library of the Royal Society in London I found a handwritten document that appears to be a translation* into English of the treatise of 1696 by Guillaume-François-Antoine Marquis de l'Hospital (1661-1704) on the Analysis of infinitesimal small quantities to describe curved lines. ${ }^{105}$ This was the first textbook that was published on the differential calculus and was based on lectures by Jean Bernoulli (1667-1748), whom l'Hospital had hired as his tutor. Assuming that Hooke received and read l'Hospital's textbook, he must have understood the significance of this mathematical development, which Newton did not discuss in any detail in his Principia. Hooke also may have been familiar with Leibniz's work on the differential calculus of $1684,{ }^{106}$ but this is unknown, because Hooke's diaries during the crucial period 1682-1687 have been lost. I also did not find evidence among Hooke's manuscripts at the Royal Society that he investigated orbital motion during this period. The differential calculus, of course, was precisely the mathematical tool that Hooke had been lacking, but he unfortunately had gone to Newton for mathematical help. One might speculate that had Hooke corresponded with Leibniz or Huygens rather than with Newton in 1679, the development of orbital dynamics might have taken a different path.

Among Hooke's unpublished manuscripts in the Wren Library at Trinity College, Cambridge, there is a memorandum entitled "A True state of the Case and Controversy between Sr Isaac Newton \& Dr Robert Hooke as to the Priority of that Noble Hypothesis of Motion of ye Planets about ye Sun as their Centre." 107 In it Hooke accurately recounts his main contributions to the theory of mechanics and gravitation that he had communicated to Newton in his correspondence in 1679 , but he does not mention his unpublished graphical computation of 1685 and related work. Hooke's memo-

* The handwriting is that of Charles Hayes, a self-taught mathematician who wrote a textbook on the differential calculus under the title, $A$ treatise on fluxions, that was based in part on l'Hospital's textbook; D.T.Whiteside, private communication, 1993. 
randum makes it clear that he understood quite well the extent and significance of his contributions to celestial mechanics relative to those of Newton. After Hooke's death in 1703, Richard Waller pencilled in the margin of the manuscript of Hooke's diary the enigmatic comment that, "Dr. Hook who was as I could prove were it a proper time the first Inventor or if you please first Hinter of those things about which Magni Nominis Heroes have contested for priority." 108 Many documents of Hooke's contributions have been lost, some of which may have pertained to his work on orbital dynamics. ${ }^{109}$ But as I have shown, we already have ample evidence that shows that the early development of modern orbital dynamics was due both to Hooke's as well as to Newton's contributions, and to their inadvertent collaboration through their timely and momentous correspondence in 1679 .

\section{Appendix}

Newton's diagram (figure 2) in his letter to Hooke of December 13,1679, appears to be a freehand sketch of the orbit of a body supposed to be moving under the action of a central force that is independent of the distance from the center $\mathrm{C}$, but it cannot be, because the initial segment of the orbit $A F O G H$ is almost exactly symmetric under reflection - a symmetry that Newton must have discovered and we now know is due to time-reversal invariance.

This symmetry suggests how Newton might have constructed his diagram. Thus, if he had a theoretical method to calculate the segment of the orbit $A F O$ from the farthermost point $A$ from the center of force at $C$ to its nearest point $O$, he could have used this segment as a template to obtain continuations of the orbit by sequential mirror reflections. One then would expect that the segments $A F O$ and $O G H$ are mirror images with respect to the line $O C$. This is not the case, however, because under a reflection and rotation that leaves the segment $A F O G H$ unchanged, the image of $C$ shifts by a small amount into the first quadrant $A C B$ while the image of $O$ shifts toward the point $F$ on the curve $A F O$. Thus, it is not clear where the center of force $C$ then is, and whether Newton had calculated the segment $A F O$ or the segment $O G H$ to use as a template to obtain continuations of the orbit by mirror reflections.

In an earlier article, ${ }^{110}$ I assumed that Newton used the segment $O G H$, and not $A F O$, as the template, because the former reproduced more accurately the correct orbit of a body in a central field of force. When the segment $A F O$ is reflected about the line $O C$, in fact, the farthermost point $A$ does not coincide with point $H$, as it should, but instead lies beyond it, as shown in figure 11, so that the reflected segment $O F A$ does not coincide with the original segment $O G H$. John Faulkner recently point out to me (private communication, June 2003) that the reflection of $A$ lies very close to the margin of Newton's letter, which suggests an alternate explanation for the shift of the image of the center $C$ noted above in Newton's diagram. To avoid having to redraw his diagram, Newton may have decided to move the image of $A$ away from the margin by shifting the reflected segment $O F A$ in such a way that its continuation with the original segment $A F O$ produces a smooth curve. I reproduced this shift, as shown in figure 12 , and found that the resulting curve agrees very well with the original segement $A F O G H$, accounting also for the superimposed lines that Newton drew along the seg- 


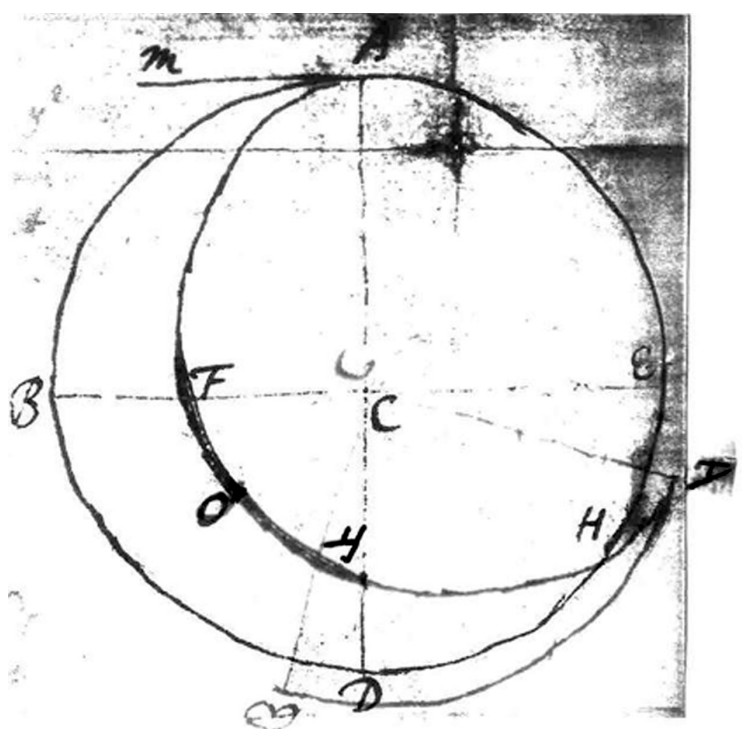

Fig. 11. Newton's diagram (figure 2) showing only the segment $A F O$ of the orbit and its mirror reflections with respect to the line $O C$ - the arc $A B$ and the lines $A C$ and $B C$.

ment $F O G$ (see figure 2) to cover the overlap of $A F O$ with its shifted image. Moreover, as I showed earlier, ${ }^{111}$ the closed curve $A F O G H$ in Newton's diagram is not a circle (although it always has been reproduced as such in the past). Indeed, while the orthogonal axes $C A$ and $C B$ are of equal length, as are the orthogonal axes $C D$ and $C E$, which also are equal to $C H$, but the shift makes $C H$ smaller than $C A$, so that the arcs $A B$ and $D E$ are sections of a circle, while the arcs $B D$ and $E A$ are sections of ellipses - a distortion that is very small owing to their very small eccentricities and hence has not been noticed earlier and is generally neglected in the literature. As a result of shifting the segment $F O H$, Newton also had to modify the next two segments $H I K$ and $K L$, which are supposed to be (but only appoximately) images of $A F O G H$ and $H I K$ relative to the lines $\mathrm{CH}$ and $\mathrm{CK}$, respectively.

If, therefore, Newton used the segment $A F O$ as a template, then he made a considerable error in calculating this segment. The angle $A C O$ in his drawing is $130^{\circ}$, while it should be approximately $104^{\circ}$, as Pelseneer pointed out ${ }^{112}$. Later, in a Scholium in a draft of his Principia, ${ }^{113}$ Newton gave this value as $110 \mathrm{o}$, which is in close agreement to the value of $107^{\circ}$ that I calculated on the basis of Newton's graphical-curvature method for computing orbits. ${ }^{114}$ Newton did not publish this Scholium in his Principia, however, because by 1686 he had found an analytic expression for this angle for orbits of small eccentricity, which he presented in Book 1, Proposition 43.

The above analysis of Newton's diagram provides further evidence that by the time of his correspondence with Hooke in 1679 Newton had not discovered the origin of Kepler's area law or the conservation of angular momentum. This conservation law 


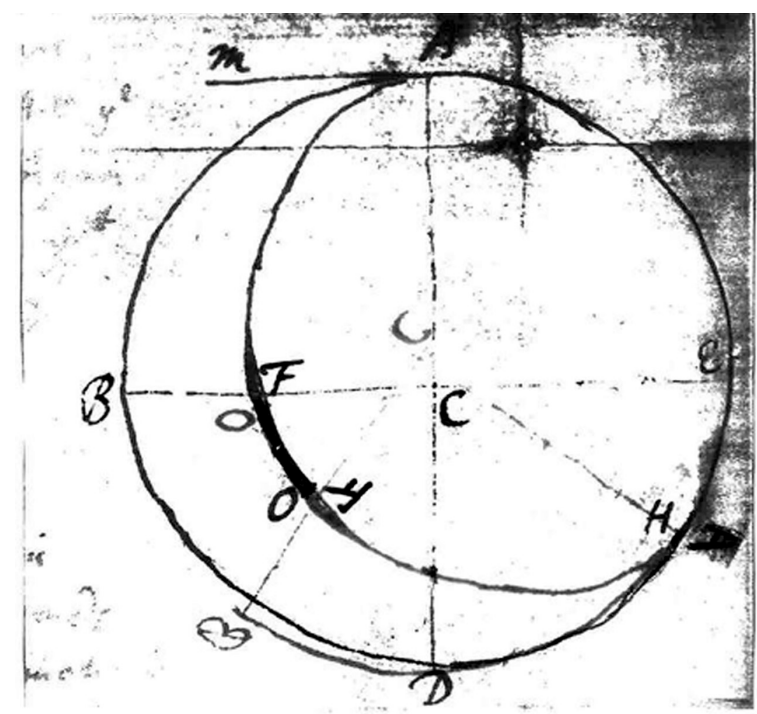

Fig. 12. Newton's diagram (figure 2) with the reflected images of the segment $A F O$ - the $\operatorname{arc} A B$ and the lines $A C$ and $B C$-now shifted so that the reflected point $A$ coincides with the original point $H$.

would have allowed him to express the velocity of a body along its orbit in terms of its position, as required by his curvature method, and to solve the outstanding problem that he had indicated by his cryptic remark of 1664 , "if its motion [velocity] in that point bee given...." 115 Instead, as I have shown, ${ }^{116}$ to determine the change in velocity of a body along its orbit, Newton would have had to resort to a further approximation. Apparently, he implemented this approximation poorly, perhaps because he was too much in a hurry to respond to Hooke, who had embarrassed him at a meeting of the Royal Society by exposing an error in his previous letter of November 28, 1679. However, as the cancelled Scholium to his Principia reveals, by 1684 Newton had obtained a good approximation to the angle $A C O$, presumably by obtaining the change in velocity of a body along its orbit by applying Kepler's area law - which he had discovered only after expressing the physical principles of orbital dynamics in mathematical form after he had assimilated Hooke's insight of "compounding the celestiall motions of the planetts of a direct motion by the tangent $\&$ an attractive motion towards the centrall body." ${ }^{117}$ (see page 21).

\section{Acknowledgments}

I thank Bernard Cleyet for his help in obtaining the stroboscopic picture of the trajectory of a ball revolving inside an inverted cone (figure 10). I also thank Malcolm MacGregor, Curtis A. Wilson, and Alan E. Shapiro for helpful comments on a draft of my paper, and Roger H. Stuewer for his thoughtful and careful editorial work on it, which included providing additional references and the biographical notes in the footnotes. 


\section{References}

1 Isaac Newton, Philosophiae Naturalis Principia Mathematica (London: 1687; reprinted London: William Dawson \& Sons, undated); revised second edition, 1713; third edition, 1726; third edition translated by Andrew Motte, The Mathematical Principles of Natural Philosophy, 2 Vols. (London: Benjamin Motte, 1729); reprinted with an introduction by I. Bernard Cohen (London: Dawsons of Pall Mall, 1968); revised by Florian Cajori, Sir Isaac Newton's Mathematical Principles of Natural Philosophy and His System of the World, 2 Vols. (Berkeley: University of California Press, 1962; reprinted New York: Greenwood Press, 1969); I. Bernard Cohen and Anne Whitman, The Principia: Mathematical Principles of Natural Philosophy: A New Translation Preceded by A Guide to Newton's Principia (Berkeley: University of California Press, 1999). In addition see I. Bernard Cohen, Introduction To Newton's “Principia” (Cambridge, Mass.: Harvard University Press, 1971); S. Chandrasekhar, Newton's Principia for the Common Reader (Oxford: Clarendon Press, 1995); Niccolò Guicciardini, Reading the Principia: The Debate on Newton's Mathematical Methods for Natural Philosophy from 1687 to 1736 (Cambridge: Cambridge University Press, 1999).

2 Michael Hunter and Simon Schaffer, ed., Robert Hooke: New Studies (Woodbridge, England and Wolfeboro, N.H.: The Boydell Press, 1989); E.N. da C. Andrade, "Wilkens Lecture. Robert Hooke," Proceedings of the Royal Society of London [A] 201 (1950), 439-473; "Robert Hooke," Scientific American 191 (December 1954), 94-98; Margaret Éspinasse, Robert Hooke (Berkeley: University of California Press, 1956).

3 Robert T. Gunther, Early Science in Oxford. Vols. VI-VII. The Life and Work of Robert Hooke (Parts I-II). Vol. VIII. The Cutler Lectures of Robert Hooke. Vol. X. The Life and Work of Robert Hooke (Part IV). Vol. XIII. The Life and Work of Robert Hooke (Part V). Micrographia, 1665 (Oxford: Oxford University Press, 1930, 1930, 1931, 1935, 1938); reprinted (London: Dawsons of Pall Mall, 1968-1969).

4 Ernst Mach, The Science of Mechanics: A Critical and Historical Account of Its Development, trans. Thomas J. McCormack (La Salle, Ill.: Open Court, 1942), p. 230.

5 W.W. Rouse Ball, An Essay on Newton's "Principia” (London and New York: Macmillan, 1893).

6 Jean Pelseneer, "Une lettre inédite de Newton," Isis 12 (1929), 237-254; Alexandre Koyré, "An Unpublished Letter of Robert Hooke to Isaac Newton," Isis 43 (1952), 312-337; reprinted in Newtonian Studies (Cambridge, Mass.: Harvard University Press, 1965), pp. 221-260.

7 F.F. Centore, Robert Hooke's Contributions to Mechanics: A Study in Seventeenth Century Natural Philosophy (The Hague: Martinus Nijhoff, 1970); I. Bernard Cohen, The Newtonian revolution: With illustrations of the transformation of scientific ideas (Cambridge: Cambridge University Press, 1980); “Newton's Discovery of Gravity,” Sci. Amer. 244 (March 1981),166-179; The Birth of a New Physics, Revised and Updated (New York and London: Norton, 1985); A. Rupert Hall, The revolution in science 1500-1750 (London and New York: Longman, 1983); Louise Diehl Patterson, "Hooke's Gravitation Theory and its influence on Newton. I. Hooke's Gravitation Theory," Isis 40 (1949), 327-341; "II. The Insufficiency of the Traditional Estimate," Isis 41 (1950), 32-45; Richard S. Westfall, "Hooke and the Law of Universal Gravitation: A Reappraisal of a Reappraisal," British Journal for the History of Science 3 (1967), 245-261; Force in Newton's Physics: The science of dynamics in the seventeenth century (London: Macdonald and New York: American Elsevier, 1971); Never at Rest: A Biography of Isaac Newton (Cambridge: Cambridge University Press, 1980); Derek T. Whiteside, "Newton's Early Thoughts on Planetary Motion: A Fresh look," Brit. J. Hist. Sci. 2 (1964), 118-137; "Before the Principia: The Maturing of Newton's Thoughts on Dynamical Astronomy, 1664-1684," Journal for the History of Astronomy 1 (1970), 5-19; "The Mathematical Principles Underlying Newton's Principia Mathematica,” J. Hist. Astron. 1 (1970),116-138.

8 René Dugas, Mechanics in the Seventeenth Century (From the Scholastic Antecedents to Classical Thought), trans. Freda Jacquot (Neuchatel: Éditions du Griffon and New York: Central Book Company, 1958), pp. 353-372; A History of Mechanics, trans. J.R. Maddox (Neuchatel: Éditions du Griffon and New York: Central Book Company, 1955), p. 216.

9 Robert Hooke, "De Potentia Restitutiva, or of Spring Explaining the Power of Springing Bodies" [Sixth Cutler Lecture, published in 1678], in Gunther, Early Science. Vol. VIII. Cutler Lectures (ref. 3), pp. 331-356. 
10 Jim Bennett, Michael Cooper, Michael Hunter, Lisa Jardine, London's Leonardo: The Life and Work of Robert Hooke (Oxford: Oxford University Press, 2003); Michael Cooper, "A More Beautiful City": Robert Hooke and the Rebuilding of London after the Great Fire (Stroud, Gloucestershire: Sutton Publishing, 2003); Ellen T. Drake, Restless Genius: Robert Hooke and his earthly thoughts (Oxford: Oxford University Press, 1996); Ofer Gal, Meanest foundations and nobler superstructures: Hooke, Newton and the compounding of the celestiall motions of the planetts (Dordrecht and Boston: Kluwer, 2002); Stephen Inwood, The Man Who Knew Too Much: The Strange and Inventive Life of Robert Hooke, 1673-1703 (London: Macmillan, 2002); Lisa Jardine, The Curious Life of Robert Hooke: the Man who Measured London (New York: HarperCollins, 2003); Richard Nichols, The Diaries of Robert Hooke, The Leonardo of London, 1635-1703 (Sussex: The Book Guild, 1994); Patri J. Pugliese, "The Scientific Achievements of Robert Hooke: Method and Mechanics," Ph.D. Thesis, Harvard University, 1982.

11 Hooke to Newton, November 24, 1679, in H.W. Turnbull, ed., The Correspondence of Isaac Newton. Vol. II. 1676-1687 (Cambridge: Cambridge University Press, 1960), p. 297.

12 Westfall, Never at Rest (ref. 7), p. 383.

13 Newton to Halley, June 20, 1679, in Turnbull, Correspondence (ref. 11), pp. 435-437.

14 Quoted in Cohen, Introduction To Newton's "Principia” (ref. 1), p. 293; see also Johannes Lohne, "Hooke versus Newton: An Analysis of the Documents in the Case on Free Fall and Planetary Motion," Centaurus 7 (1960), 6-52; on 48-49.

15 Michael Nauenberg, "Newton's Early Computational Method for Dynamics," Archive for History of Exact Sciences 46 (1994), 221-252.

16 Michael Nauenberg, "Hooke, orbital motion, and Newton's Principia," American Journal of Physics 62 (1994), 331-350; “On Hooke's 1685 Manuscript on Orbital Mechanics," Historia Mathematica 25 (1998), 89-93. See also Patri J. Pugliese, "Robert Hooke and the Dynamics of Motion in a Curved Path," in Hunter and Schaffer, Hooke (ref. 2), pp. 200-204.

17 Isaac Newton, De Motu Corporum in Gyrum [1684]; facsimilie in D.T. Whiteside, ed., The preliminary manuscripts for Isaac Newton's 1687 Principia, 1684-1685 (Cambridge: Cambridge University Press, 1989), pp. 3-11, with subsequent parts, pp. 12-33; trans. with notes John Herivel, The Background to Newton's Principia: A Study of Newton's Dynamical Researches in the Years 1664-84 (Oxford: Clarendon Press, 1965), pp. 277-292; for discussion, see D.T. Whiteside, "The Prehistory of the Principia from 1664 to 1686," Notes and Records of the Royal Society of London 45 (1991), 11-61.

18 René Descartes, Principles of Philosophy, trans. Valentine R. Miller and Reese P. Miller (Dordrecht, Boston, and London: D. Reidel, 1983), plate ii, p. 290.

19 Christiaan Huygens, "De Vi Centrifuga [1659]," in Société Hollandaise des Sciences, ed., Oeuvres Complètes de Christiaan Huygens, Vol. XVI. Percussion, etc. (La Haye: Martinus Nijoff, 1929), pp. 253-301. For Newton see Herivel, Background (ref. 17), pp. 192-198, and A. Rupert Hall, "Newton on the Calculation of Central Forces," Annals of Science 13 (1957), 62-71.

20 Nauenberg, "Newton's Early Computational Method" (ref. 15).

21 Quoted in J. Bruce Brackenridge, The Key to Newton's Dynamics: The Kepler Problem and the Principia (Berkeley: University of California Press, 1995), p. 63; also quoted with some variations in Herival, Background (ref. 17), p. 130.

22 Newton to Hooke, December 13, 1679, in Turnbull, Correspondence (ref. 11), pp. 307-308.

23 Nauenberg, "Newton's Early Computational Method" (ref. 15).

24 Newton to Crompton, April 1681[?], in Turnbull, Correspondence (ref. 11), p. 361.

25 Nauenberg, "Newton's Early Computational Method"(ref. 15); J. Bruce Brackenridge and Michael Nauenberg, "Curvature in Newton's dynamics," in I. Bernard Cohen and George E. Smith, ed., The Cambridge Companion to Newton (Cambridge and New York: Cambridge University Press, 2002), pp. 231-260.

26 Michael Nauenberg, "Newton's perturbation methods for three-body problem and their application to Lunar motion," in Jed Z. Buchwald and I.Bernard Cohen, ed., Isaac Newton's Natural Philosophy (Cambridge, Mass. and London:The MIT Press, 2001), pp. 189-224; “Newton's Portsmouth Perturbation Method and its Application to Lunar Motion," in Richard H. Dalitz and Michael Nauenberg, ed., The Foundations of Newtonian Scholarship (Singapore: World Scientific, 2000), pp. 157-194. 
27 Brackenridge, Key (ref. 21), pp. 217-218; Chandrasekhar, Newton's Principia (ref. 1), p. 115. Without justification Chandrasekhar claims that "these simple relations must have escaped him [Newton] during the two years when he was under extreme pressure of writing the Principia; and that they became transparent to him during his 'lonely voyages,' in the leisure of his London years."

28 Nauenberg, "Newton's Early Computational Method” (ref. 15).

29 Michael Nauenberg, "Kepler's area law in the Principia: Filling in some details in Newton's proof of Proposition 1," Historia Mathematica 30 (2003), 441-456.

30 Reproduced in Whiteside, "Prehistory" (ref. 17), p. 13.

31 Quoted in Cohen and Whitman, The Principia (ref. 1), p. 444.

32 Richard Waller, "The Life of Dr. Robert Hooke," in Gunther, Early Science. Vol. VI. Hooke (Part I) (ref. 3), pp. 1- 68; on 67; also in Richard Waller, The Posthumous Works of Robert Hooke, M.D. S.R.S. Geom. Prof. Gresh. \&c, Containing his Cutlerian Lectures, and other Discourses, Read at the Meetings of the Illustrious Royal Society, Second Edition With a New Introduction by T.M. Brown (London: Frank Cass, 1971), pp. i-xxvii; on p. xxviii.

33 Robert Hooke, An Attempt To prove the Motion of the Earth from Observations (London: Printed by T.R. for John Martyn Printer to the Royal Society at the Bell in St. Pauls Church-yard, 1674); reprinted in Gunther, Early Science. Vol. VIII. Cutler Lectures (ref. 3), pp. 1-28; reported in Philosophical Transactions 9 (1674), 12-13.

34 Robert Hooke, "A Discourse of the Nature of Comets [1682]," in Waller, Posthumous Works (ref. 32), pp. 184-185.

35 Patterson, "Hooke's Gravitation Theory" (ref. 7).

36 Newton to Halley, June 20, 1686, in Turnbull, Correspondence (ref. 11), p. 438.

37 Halley to Newton, May 22, 1686, in Turnbull, Correspondence (ref. 11), p. 431.

38 Nauenberg, "Newton's Early Computational Method" (ref. 15).

39 Quoted in Cajori, Newton's Mathematical Principles (ref. 1), p. 550.

40 Lohne, "Hooke versus Newton" (ref. 14); V.I Arnol'd, Huygens and Barrow, Newton and Hooke: Pioneers in mathematical analysis and catastrophe theory from evolvents to quaisicrystals, trans. Eric J.F. Primrose (Basel: Birkhäuser, 1990).

41 Hall, revolution in science (ref. 7), p. 303

42 Ibid., p. 314

43 Quoted in French in Rouse Ball, Essay on Newton's Principia (ref. 5), p. 69: "servent à faire voir quelle distance il y a entre une verité entrevue et une verité demontreé...." Rouse Ball cited as his source Stephen Peter Rigaud, Historical Essay on the First Publication of Sir Isaac Newton's Principia (Oxford: Oxford University Press, 1838), p. 66, which has been reprinted with an Introduction by I. Bernard Cohen (New York and London: Johnson Reprint Corporation, 1972), p. 66

44 Robert Hooke, "Motion in a Curve. A Statement of Planetary Movements as a Mechanical Problem [1666]," in Gunther, Early Science. Vol. VI. Hooke (Part I) (ref. 3), pp. 265-268.

45 Hooke, "Discourse" (ref. 34), p. 179.

46 Ibid., p. 167.

47 Pugliese, "Hooke" (ref. 16), pp. 181-205; on p. 202.

48 Newton, De Motu; facsimile in Whiteside, preliminary manuscripts (ref. 17), pp. 3-11.

49 Pugliese, "Hooke" (ref. 16), pp. 203, 200, 204.

50 Nauenberg, "Hooke, orbital motion" (ref. 16), pp. 331-350.

51 Ibid.

52 Flamsteed to Newton, December 27, 1684, in Turnbull, Correspondence (ref. 11), p. 405.

53 Halley to Newton, June 29, 1686, in Turnbull, Correspondence (ref. 11), p. 442.

54 Quoted in Herival, Background (ref. 17), p. 278.

55 Ibid.

56 Quoted in Nauenberg, "Hooke, orbital motion" (ref. 16), p. 347.

57 Hooke, "Statement of Planetary Movements [1666]" (ref. 44), pp. 265-267; also in Thomas Birch, The History of the Royal Society of London for Improving of Natural Knowledge from Its First Use. Vol. 2 (London: A. Millar, 1756; reprinted New York and London: Johnson Reprint Corporation, 1968), pp. 90-92.

58 Quoted in Gunther, Early Science, Vol. VI. Hooke (Part I) (ref. 3), p. 265, and in Birch, History (ref. 57), p. 91. 
59 Angus Armitage, “Borell's Hypothesis' and the Rise of Celestial Mechanics,” Ann. Sci. 6 (1950), 268-282; Hall, revolution in science (ref. 7), pp. 302-304.

60 Hooke, "Statement of Planetary Movements [1666]" (ref. 44), p. 266

61 Ibid., p. 267.

62 Ibid.

63 Ibid.

64 Hooke to Newton, December 6, 1679, in Turnbull, Correspondence (ref. 11), pp. 304-306; figure and quotation on p. 305 .

65 Ibid., p. 305.

66 Nauenberg, "Hooke, orbital motion" (ref. 16).

67 Hooke, "Gravity," in Gunther, Early Science, Vol. VI. Hooke (Part I) (ref. 3), pp. 256-259.

68 Hooke, Attempt (ref. 33), p. 2.

69 Gunther, Early Science, Vol. VI. Hooke (Part I) (ref. 3), p. 343.

70 Hooke, Attempt (ref. 33), pp. 1-28; reported in Philosophical Transactions 9 (1674), 12-13.

71 Hooke, Attempt (ref. 33), pp. 27-28.

72 Gunther, Early Science. Vol. VI. Hooke (Part I) (ref. 3), pp. 22-23.

73 Cooper, "Beautiful City" (ref. 10).

74 Hooke to Newton, November 24, 1679, in Turnbull, Correspondence (ref. 11), p. 297.

75 Pelseneer, "Une lettre" (ref. 6), p. 244.

76 Hooke to Newton, January 6, 1680, in Turnbull, Correspondence (ref. 11), p. 309.

77 Ibid.

78 Ibid.

79 Ibid.

80 Hooke to Newton, January 17, 1680, in Turnbull, Correspondence (ref. 11), p. 313.

81 Newton to Hooke, November 28, 1679, in Turnbull, Correspondence (ref. 11), pp. 300, 302.

82 Ibid., p. 301.

83 Hooke to Newton, November 24, 1679, in Turnbull, Correspondence (ref. 11), p. 298.

84 Hooke, Attempt (ref. 33).

85 Newton to Halley, July 27, 1686, in Turnbull, Correspondence (ref. 11), pp. 446-447.

86 Robert Hooke, “A True state of the Case and Controversy between Sr Isaac Newton \& Dr Robert Hooke as to the Priority of that Noble Hypothesis of Motion of ye Planets about ye Sun as their Centre," undated manuscript, Trinity College Library, Cambridge; reprinted in Gunther, Early Science, Vol. X. Hooke (Part IV) (ref. 3), pp. 56-60; on p. 60.

87 Hooke, Attempt (ref. 33).

88 Henry Oldenburg (1618-1677), Secretary of the Royal Society, published extracts of Huygens's and Cassini's letters commenting on Hooke's tract in Philosophical Transactions 9 (1674), 91-92. Newton's correspondence with Hooke and Halley makes clear that he also had become familiar with Hooke's tract.

89 Quoted in Gunther, Early Science, Vol. X. Hooke (Part IV) (ref. 3), p. 98. I thank Niccolò Guicciardini for translating the Latin into English.

90 Hooke to Newton, January 6, 1680, in Turnbull, Correspondence (ref. 11), p. 309.

91 Ibid.

92 Newton to Halley, June 20, 1686, in Turnbull, Correspondence (ref. 11), pp. 435-436.

93 For example, ibid., pp. 435-440.

94 Halley to Newton, June 29, 1686, in Turnbull, Correspondence (ref. 11), pp. 441-445.

95 Newton to Halley, July 14, 1686, in Turnbull, Correspondence (ref. 11), pp. 444-445.

96 Newton to Halley, July 27, 1686, in Turnbull, Correspondence (ref. 11), p. 447.

97 Newton to Hooke, December 13, 1679, in Turnbull, Correspondence (ref. 11), p. 308.

98 Nauenberg, "Newton's Early Computational Method" (ref. 15). For Newton's work, see D.T. Whiteside, ed., The Mathematical Papers of Isaac Newton. Vol. I. 1664-1666 (Cambridge: Cambridge University Press, 1967), pp. 252-255; Vol. III. 1670-1673 (Cambridge: Cambridge University Press, 1969), pp. 151-159.

99 Quoted in Cohen, Introduction To Newton's "Principia” (ref. 1), p. 293.

100 Halley to Newton, June 29, 1686, in Turnbull, Correspondence (ref. 11), p. 442.

101 Ibid. 
102 Nauenberg, "Hooke, orbital motion" (ref. 16).

103 Ibid.; see also Nauenberg, "Hooke's 1685 Manuscript" (ref. 16).

104 Hooke, "Discourse" (ref. 34), pp. 184-185.

105 Guillaume François Marquis de l'Hospital, Analyse des infiniment petits pour l'intelligence des lignes courbes (Paris: l'Imprimerie Royale, 1696).

106 Gottfried Leibniz, "Nova methodus pro maximis et minimis, itemque tangentibus, quae nec fractas nec irrationales quantitates moratur, et singulare pro illis calculi genus," Acta Eruditorum [Leipzig] 10 (1684), 467-473; reprinted in C.I. Gerhardt, Leibnizens mathematische Schriften. Band I, zweite Abtheilung (Halle: H.W. Schmidt, 1858), pp. 220-226; reprinted Mathematische Schriften, Band V (Hildesheim and New York, Georg Ohms Verlag, 1971), pp. 220-226.

107 Hooke, "True state of the Case and Controversy" (ref. 86), pp. 56-60.

108 Quoted in Patterson, "Hooke's Gravitational Theory. I" (ref. 7), p. 329.

109 Ibid.

110 Nauenberg, "Newton's Early Computational Method" (ref. 15).

111 Ibid.

112 Pelseneer, "Une lettre" (ref. 6).

113 Whiteside, Mathematical Papers (footnote p, p. 21 [CHANGE THIS IN PRINTED PAPER] ), pp. 149-152; on p. 151.

114 Nauenberg, "Newton's Early Computational Method" (ref. 15).

115 Quoted in Brackenridge, Key (ref. 21), p. 63.

116 Nauenberg, "Newton's Early Computational Method" (ref. 15).

117 Hooke to Newton, November 24, 1679, in Turnbull, Correspondence (ref. 11), p. 297.

Department of Physics

University of California

Santa Cruz, CA 95064 USA

e-mail: michael@mike.ucsc.edu 